\title{
Plasma Dynamics in Low-Electron-Beta Environments
}

\author{
Stanislav Boldyrev ${ }^{1,2 *}$, Nuno F. Loureiro ${ }^{3}$ and Vadim Roytershteyn ${ }^{2}$ \\ ${ }^{1}$ Department of Physics, University of Wisconsin-Madison, Madison, WI, United States, ${ }^{2}$ Center for Space Plasma Physics, \\ Space Science Institute, Boulder, CO, United States, ${ }^{3}$ Plasma Science and Fusion Center, Massachusetts Institute of \\ Technology, Cambridge, MA, United states
}

Recent in situ measurements by the MMS and Parker Solar Probe missions bring interest to small-scale plasma dynamics (waves, turbulence, magnetic reconnection) in regions where the electron thermal energy is smaller than the magnetic one. Examples of such regions are the Earth's magnetosheath and the vicinity of the solar corona, and they are also encountered in other astrophysical systems. In this brief review, we consider simple physical models describing plasma dynamics in such low-electron-beta regimes, discuss their conservation laws and their limits of applicability.

Keywords: collisionless plasma, magnetic fields, heliosphere, solar wind, solar corona, earth magnetosheath, earth magnetosphere, plasma turbulence

\section{OPEN ACCESS}

Edited by:

Alessandro Retino,

UMR7648 Laboratoire de physique des plasmas (LPP), France

Reviewed by: Francesco Malara,

University of Calabria, Italy

Mikhail V. Medvedev,

University of Kansas, United States

*Correspondence: Stanislav Boldyrev boldyrev@wisc.edu

Specialty section:

This article was submitted to Space Physics,

a section of the journal Frontiers in Astronomy and Space

Sciences

Received: 24 October 2020 Accepted: 25 January 2021

Published: 05 May 2021

Citation:

Boldyrev S, Loureiro NF and Roytershteyn V (2021) Plasma

Dynamics in Low-ElectronBeta Environments.

Front. Astron. Space Sci. 8:621040. doi: 10.3389/fspas.2021.621040

\section{INTRODUCTION}

Astrophysical plasmas (e.g., the Interstellar medium, solar wind, etc) are often in a state of a rough equipartition between the kinetic energies of the particles and the energy of the magnetic fields. However, there are important astrophysical and space environments, such as the Earth's magnetosphere and magnetosheath, and the solar corona and its vicinity, that are characterized by low electron plasma beta, that is, low ratio of electron thermal to magnetic energy, $\beta_{e}=8 \pi n_{e} T_{e} / B^{2}$ (e.g., Cranmer et al., 2009; Štverák et al., 2015; Bale et al., 2016; Chen et al., 2014), where $n_{e}$ and $T_{e}$ are the electron density and temperature, and $B$ is the magnetic field strength. Such plasmas are also nearly collisionless in that the characteristic times of turbulent fluctuations are much shorter than the Coulomb collision times. The interest in plasma dynamics in low-beta regimes is also fueled by recent in situ measurements by NASA's MMS and Parker Solar Probe missions, as well as by the measurements expected from the Solar Orbiter spacecraft (e.g., Phan et al., 2018; Chen et al., 2020; Bale et al., 2019; Kasper et al., 2019). In this contribution we briefly review the theoretical frameworks for studying collisionless low-electron-beta plasma dynamics.

In a weakly collisional plasma, the electrons and the ions do not exchange energy efficiently due to the strong difference in their masses. Therefore, it is a common situation that the ion temperature is different from the electron one. In our treatment of the problem we will, therefore, distinguish between the ion and electron betas $\beta_{s}=8 \pi n_{s} T_{s} / B^{2}$, where $s=\{e, i\}$. While we will concentrate on the case of small electron beta $\beta_{e} \ll 1$, we will not necessarily assume the same for the ion beta, and will consider the cases of $\beta_{i} \ll 1$ as well as $\beta_{i} \sim 1$. For example, the Earth's magnetosphere is characterized by $\beta_{i} \ll m_{e} / m_{i}, \beta_{e} \ll m_{e} / m_{i}$, the solar corona and its vicinity correspond to $\beta_{e} \lesssim 0.01$ and $\beta_{i} \lesssim 0.1$, the Earth's magnetosheath $\beta_{e} \sim 0.1, \beta_{i} \leq 1$. Other environments with low electron beta include downstream regions of collisionless shocks and magnetospheres of accretion discs (e.g., Quataert, 1998; Vink et al., 2015; Ghavamian et al., 2013).

The most rigorous treatment of a collisionless plasma is provided by the kinetic framework. However, kinetic framework presents considerable challenges for theoretical and especially numerical treatments (but see some examples in e.g. (Schekochihin et al., 2009; Servidio et al., 
2012; Valentini et al., 2017; Grošelj, 2019; Roytershteyn et al., 2019; Franci et al., 2020)). In many important cases, a simplified fluid-like description is possible that is much more physically transparent and allows for efficient numerical studies of plasma waves, turbulence, magnetic reconnection, structure formation, etc. The derivations of such simplified models can be performed using various approaches (reduced two-fluid, gyrofluid, gyrokinetic, kinetic, etc.), and such derivations are scattered in the literature. In this brief review, we discuss several models which we believe are relevant for the above mentioned space physics applications. Our goal is to present a unifying physical derivation of the governing equations, describe the corresponding conservation laws, and discuss the limits of applicability of each of the models. We hope our presentation will be useful for space physicists or astrophysicists who are not necessarily experts in plasma physics.

\section{Model Equations}

In this section we present a general derivation of the model equations, and then consider the limits of $\beta_{i}$ and $\beta_{e}$ mentioned in the introduction. As it is generally the case in magnetized plasma turbulence, we assume the presence of a uniform magnetic field (the guide field), which mimics the magnetic field of external sources (i.e., magnetospheric field) or the magnetic field generated by large-scale turbulent motions. At small scales, the magnetic fluctuations are small, so we separate them from the guide field $\mathbf{B}=B_{0} \widehat{\boldsymbol{z}}+\delta \mathbf{B}$. ${ }^{1}$ We consider the case of small electron beta, so it will be easy to start with the equations describing the electron dynamics where we can neglect the effects related to the electron gyroradius. There are analytical and observational reasons to believe that smallscale fluctuations are oblique in that their wavenumbers along the guide field are much smaller than the wavenumbers in the perpendicular direction, $k_{\|} \ll k_{\perp}$ (e.g., Shebalin et al., 1983; Chen, 2016). Moreover, in the case of strong developed turbulence, the magnetic fluctuations tend to approach the so-called critical balance state (e.g., Goldreich and Sridhar, 1995; Perez and Boldyrev, 2010), which can be expressed by the following self-consistent ordering of the perturbation parameters,

$$
k_{\|} / k_{\perp} \sim|\delta \mathbf{B}| / B_{0} \sim \delta n / n_{0} \ll 1 .
$$

Our general approach in this section is similar to that adopted in (e.g., Chen and Boldyrev, 2017; Milanese et al., 2020), while more refined derivations can be found in (Passot et al., 2017; Passot et al., 2018) where finite Larmor radius corrections are taken into account. In a collisionless plasma, the electron gyro orbits drift in the field-perpendicular direction. The modes we are interested in

\footnotetext{
${ }^{1}$ Such a set up is an approximation based on two properties that are believed to be characteristic of strong magnetic turbulence. First is the locality of turbulence, implying that significant nonlinear interaction occurs among fluctuations of comparable scales. Second is the observation that the dynamics at a given small scale are mediated by the presence of a guide magnetic field. However, the strongest magnetic fluctuations are provided by the largest eddies, therefore, such a magnetic field is almost uniform at the small scales of interest.
}

have frequencies that are much lower than the electron cyclotron frequency $\Omega_{e}$. To the zeroth and first orders in the small parameter $\omega / \Omega_{e}$, this motion consists of the standard $E \times B$ drift and the polarization drift,

$$
\mathbf{v}_{\perp}=\mathbf{v}_{E}-\frac{m_{e} c}{e B^{2}} \mathbf{B} \times \frac{d_{E}}{d t} \mathbf{v}_{E}
$$

where $\mathbf{v}_{E}=c(\mathbf{E} \times \mathbf{B}) / B^{2}$ is the $E \times B$ drift, the total time derivative is $d_{E} / d t \equiv \partial / \partial t+\mathbf{v}_{E} \cdot \nabla$, and $e$ is the modulus of the electron charge. (Obviously, for $\omega \ll \Omega_{i}$ an equation similar to Eq. 2 can be written for the ions as well.) In the zeroth-order term (the $\mathbf{v}_{E}$ velocity) we need to substitute the magnetic field expanded up to the first order, that is, $\mathbf{B}=\mathbf{B}_{0}+\delta \mathbf{B}$ and $B^{2} \approx B_{0}^{2}+2 B_{0} \delta B_{z}$, while in the polarization drift (the second term in Eq. 2) we keep only the zeroth-order magnetic field. The magnetic field does not constrain the electron motion in the field-parallel direction, so that the fluctuating parallel electric field will drive the electric current $J_{\|}$. It is easy to see, however, that due to their large masses, the ions will respond to the fluctuating electric field with much smaller velocities, so that the current will be dominated by the electrons, $J_{\|}=-n e v_{\|}$. Since, due to small fluctuations, the magnetic-field lines deviate from the $z$-direction only slightly, the field-parallel components of the vector fields are very close to their $z$-components, i.e., $J_{\|} \approx J_{z}$. This, however, is not true for nearly field-perpendicular wave vectors, so that $k_{\|} \neq k_{z}$. For this reason, the gradient in the fieldparallel direction will be given to the first order in magnetic field fluctuations by

$$
\nabla_{\|}=\frac{\mathbf{B}}{B} \cdot \nabla=\partial / \partial z+\left(\delta \mathbf{B}_{\perp} / B_{0}\right) \cdot \nabla
$$

which is also consistent with the adopted ordering (1). In the same approximation, the field-perpendicular gradients are the same as gradients in the horizontal coordinate plane, $\nabla_{\perp}=$ $(\partial / \partial x, \partial / \partial y)$

Finally, we need to relate the parallel electric current to the fluctuating magnetic and electric fields. From the AmpereMaxwell equation, we have

$$
J_{z}=-\frac{c}{4 \pi} \nabla_{\perp}^{2} A_{z}+\frac{1}{4 \pi c} \frac{\partial^{2}}{\partial t^{2}} A_{z} \approx-\frac{c}{4 \pi} \nabla_{\perp}^{2} A_{z},
$$

where $\mathbf{A}$ is the vector potential, $\delta \mathbf{B}_{\perp}=-\widehat{\mathbf{z}} \times \nabla_{\perp} A_{z}$, the Lorentz gauge is assumed for simplicity, and in the last line we neglected the time derivative of the vector potential, since $\omega \sim k_{\|} v_{A} \ll k_{\perp} c$. Here $v_{A}$ is the Alfven speed. The last condition amounts to neglecting the displacement current in the Ampere-Maxwell equation. We can now substitute $\mathrm{v}_{\perp}$ and $v_{\|}$expressed through the electric and magnetic potentials, in the electron continuity equation $\partial_{t} n_{e}+\nabla_{\perp}\left(n_{e} \mathbf{v}_{\perp}\right)+\nabla_{\|}\left(n_{e} v_{\|}\right)=0$, and get after somewhat lengthy but straightforward algebra (for a more detailed discussion we refer the reader to (Chen and Boldyrev, 2017; Milanese et al., 2020):

$$
\begin{aligned}
& \frac{\partial}{\partial t}\left(\frac{\delta n_{e}}{n_{0}}-\frac{\delta B_{z}}{B_{0}}+\frac{m_{e} c^{2}}{e B_{0}^{2}} \nabla_{\perp}^{2} \phi\right)+\frac{c}{B_{0}}\left(\widehat{z} \times \nabla_{\perp} \phi\right) \cdot \nabla_{\perp}\left(\frac{\delta n_{e}}{n_{0}}-\frac{\delta B_{z}}{B_{0}}\right. \\
& \left.+\frac{m_{e} c^{2}}{e B_{0}^{2}} \nabla_{\perp}^{2} \phi\right)=-\frac{c}{4 \pi n_{0} e} \nabla_{\|} \nabla_{\perp}^{2} A_{z},
\end{aligned}
$$


where $\phi$ is the electric potential.

In order to proceed further, we need to specify what particular limits we consider. We will do this in the following sections. Here, we simply assume that the electron and ion gyroradii are sufficiently small and we address the scales above the ion and electron gyroradii. We also assume that the frequencies of the fluctuations are much smaller than the cyclotron frequencies of the plasma species. In this case, we can write an equation analogous to Eq. 5 for the ions (by replacing $m_{e} \rightarrow m_{i}$, $e \rightarrow-e$, and neglecting $v_{\|}$in the ion equation because of ion inertia), and subtract one equation from the other. As a result, we get

$$
\begin{aligned}
& \frac{\partial}{\partial t}\left(\rho-\frac{n_{0} m_{i} c^{2}}{B_{0}^{2}} \nabla_{\perp}^{2} \phi\right)+\frac{c}{B_{0}}\left(\widehat{z} \times \nabla_{\perp} \phi\right) \cdot \nabla_{\perp}\left(\rho-\frac{n_{0} m_{i} c^{2}}{B_{0}^{2}} \nabla_{\perp}^{2} \phi\right) \\
& \quad=\frac{c}{4 \pi} \nabla_{\|} \nabla_{\perp}^{2} A_{z}
\end{aligned}
$$

where $\rho=\left(\delta n_{i}-\delta n_{e}\right) e$ is the density of the electric charge, and we assume singly charged ions. In this equation, we have neglected the electron polarization drift velocity as it is smaller than the ion one by $m_{e} / m_{i}$. By using Gauss's law $\rho=-(1 / 4 \pi) \nabla_{\perp}^{2} \phi$, and normalizing the variables as

$$
\tilde{\phi}=\phi c / B_{0}, \quad \tilde{A}_{z}=A_{z} / \sqrt{4 \pi n_{0} m_{i}},
$$

one rewrites this equation as a charge continuity equation:

$$
\frac{\partial}{\partial t} \nabla_{\perp}^{2} \phi+\left(\widehat{z} \times \nabla_{\perp} \phi\right) \cdot \nabla_{\perp} \nabla_{\perp}^{2} \phi=-\frac{v_{A}}{1+v_{A}^{2} / c^{2}} \nabla_{\|} \nabla_{\perp}^{2} A_{z}
$$

where for simplicity we have omitted the overtilde signs. In this equation, $v_{A}=B_{0} / \sqrt{4 \pi m_{i} n_{0}}$ is the Alfvén velocity and, in the normalized variables (7), the parallel gradient has the form

$$
\nabla_{\|}=\partial / \partial z-v_{A}^{-1}\left(\widehat{z} \times \nabla_{\perp} A_{z}\right) \cdot \nabla_{\perp} .
$$

The term $v_{A}^{2} / c^{2}=\Omega_{i}^{2} / \omega_{p i}^{2}$ reflects the deviation from quasineutrality of the plasma. Here, $\Omega_{i}$ is the ion cyclotron frequency and $\omega_{p i}$ is the ion plasma frequency. When this term is small, $\Omega_{i}^{2} / \omega_{p i}^{2} \ll 1$, we have $\left|\delta n_{i}-\delta n_{e}\right| \ll \delta n$, and the charge density fluctuations can be neglected in the charge continuity equation, $\partial \rho / \partial t \ll \nabla_{\perp} \cdot \mathbf{J}_{\perp}$. Interestingly, even a mild breakdown of the analogous quasineutrality condition for the electrons, $\Omega_{e}^{2} / \omega_{p e}^{2} \ll 1$, leads to a difference between the electron and ion density fluctuations, which may be significant for the plasma dynamics (e.g., Roytershteyn et al., 2019). We will assume in our consideration that the quasineutrality condition holds for both species as it is a common situation in many natural applications (obviously, it always holds better for the heavier particles). We however mention that when this condition is broken for the electrons, that is, $\Omega_{e}^{2} / \omega_{p e}^{2} \gtrsim 1$ (we will call this case the low plasma density case), our derivation has narrower limits of applicability. Indeed, from Eq. 4 for the electron parallel current, we can estimate for the electron velocity fluctuations at scale $\lambda \sim 1 / k_{\perp}$, $v_{\|, \lambda}^{2} / c^{2} \sim\left(k_{\perp} d_{e}\right)^{2}\left(\delta B_{\lambda} / \mathrm{B}_{0}\right)^{2}\left(\Omega_{e} / \omega_{\mathrm{pe}}\right)^{2} \quad$ Here $d_{e}=c / \omega_{p e}$ is the electron inertial scale. As our case is nonrelativistic, we therefore have to require

$$
\left(k_{\perp} d_{e}\right)^{2}\left(\delta B_{\lambda} / \mathrm{B}_{0}\right)^{2} \ll \omega_{\mathrm{pe}}^{2} / \Omega_{\mathrm{e}}^{2},
$$

which imposes an additional restriction on the fluctuations amplitudes and scales in the low-density case. When restriction (10) is not satisfied, we cannot neglect the relativistic effects and the displacement current, and cannot assume the ordering $k_{\|} \ll k_{\perp}$.

We need to supplement the charge continuity Eq. 8 with the equation for the parallel component of the electron velocity field, which reads

$$
\frac{\partial \boldsymbol{v}_{\|}}{\partial t}+\left(\boldsymbol{v}_{E} \cdot \nabla_{\perp}\right) \boldsymbol{v}_{\|}=-\frac{e}{m_{e}} E_{\|}-\frac{1}{m_{e} n_{0}} \nabla_{\|} p_{e} .
$$

Expressing the parallel velocity field through the electric current, and substituting for the electric field $E_{\|}=-\nabla_{\|} \phi-\partial A_{z} / \partial t$ (where we use the previously discussed approximation $A_{\|} \approx A_{z}$ ) we obtain using the same normalization for $A_{z}$ and $\phi$ as in Eq. 7,

$$
\begin{aligned}
& \frac{\partial}{\partial t}\left(1-d_{e}^{2} \nabla_{\perp}^{2}\right) A_{z}+\left(\widehat{z} \times \nabla_{\perp} \phi\right) \cdot \nabla_{\perp}\left(1-d_{e}^{2} \nabla_{\perp}^{2}\right) A_{z} \\
& \quad=-v_{A} \frac{\partial}{\partial z} \phi+\frac{d_{i}}{n_{0} m_{i}} \nabla_{\|} p_{e} .
\end{aligned}
$$

In general, there is no rigorous closure for the pressure term $p_{e}$ in hydrodynamic-type equations describing a collisionless plasma. One, however, can consider several limiting cases, when approximate expressions may be obtained.

\section{Case of $\beta_{\mathrm{e}} \ll \mathrm{m}_{\mathrm{e}} / \mathrm{m}_{\mathrm{i}}$ and $\beta_{\mathrm{i}} \ll 1$ (Cold Electrons and lons)}

First is the case of cold electrons, when the typical phase velocity of the fluctuations is larger than the thermal velocity of the electrons, $\omega / k_{\|} \gg v_{T e}$; the equations that we discuss in this section have been considered in (Loureiro and Boldyrev, 2018; Milanese et al., 2020). Assuming that the fluctuations are of the Alfvén type, this condition means that $\beta_{e} \ll m_{e} / m_{i}$. For the ions, it means $\beta_{i} \ll 1$. In this case, we may neglect the electron pressure in Eq. 12. We can therefore use the following system of equations in the case of cold plasma:

$$
\begin{gathered}
\frac{\partial}{\partial t} \nabla_{\perp}^{2} \phi+\left(\widehat{z} \times \nabla_{\perp} \phi\right) \cdot \nabla_{\perp} \nabla_{\perp}^{2} \phi=-v_{A} \nabla_{\|} \nabla_{\perp}^{2} A_{z} \\
\frac{\partial}{\partial t}\left(1-d_{e}^{2} \nabla_{\perp}^{2}\right) A_{z}+\left(\widehat{z} \times \nabla_{\perp} \phi\right) \cdot \nabla_{\perp}\left(1-d_{e}^{2} \nabla_{\perp}^{2}\right) A_{z}=-v_{A} \frac{\partial}{\partial z} \phi
\end{gathered}
$$

The linear modes supported by this system of equations have the dispersion relation

$$
\omega^{2}=\frac{k_{z}^{2} v_{A}^{2}}{1+k_{\perp}^{2} d_{e}^{2}},
$$

and are known as the inertial Alfvén modes. At large scales $k_{\perp} d_{e} \ll 1$, they turn into the magnetohydrodynamic shear Alfvén modes as the governing system (13), (14) itself turns into the reduced MHD equations (e.g., Kadomtsev and Pogutse, 1974; Strauss, 1976; Biskamp, 2003; Tobias et al., 2013). The term containing the electron inertial scale $d_{e}$ should be kept if this scale is larger than the ion gyroscale, $\rho_{i}$. Since $d_{e}^{2} / \rho_{i}^{2}=\left(m_{e} / m_{i}\right) / \beta_{i}$, the electron inertial effects are, therefore, relevant when $\beta_{i} \ll m_{e} / m_{i}$. In the opposite limit, the electron inertial terms are negligible and 
Eqs. 13, 14 turn into the reduced MHD equations in the whole range of scales $k_{\perp}^{2} \rho_{i}^{2} \ll 1$. It is interesting to point out the conservation laws of these equations, the energy and generalized helicity

$$
\begin{gathered}
E=\int\left[\left(\nabla_{\perp} A_{z}\right)^{2}+d_{e}^{2}\left(\nabla_{\perp}^{2} A_{z}\right)^{2}+\left(\nabla_{\perp} \phi\right)^{2}\right] d^{3} x, \\
H=\int\left[\nabla_{\perp}^{2} \phi\left(1-d_{e}^{2} \nabla_{\perp}^{2}\right) A_{z}\right] d^{3} x .
\end{gathered}
$$

The generalized helicity conservation law for this case has been considered in Loureiro and Boldyrev (2018) and Milanese et al. (2020). The latter paper also discusses its nontrivial role in the turbulent energy cascade at kinetic scales $k_{\perp} d_{e}>1$, in particular, in establishing the so-called dynamic phase alignment of magnetic and velocity fluctuations at small scales.

\section{Case of $m_{\mathrm{e}} / \mathbf{m}_{\mathrm{i}} \ll \beta_{\mathrm{e}} \ll 1$ and $\beta_{\mathrm{i}} \ll 1$ (Hot Electrons, Cold Ions)}

In the considered limit, the systems of equations have been derived in e.g., (Camargo et al., 1996; Terry et al., 2001; Boldyrev et al., 2015). In this case the electrons are hot in that their thermal velocity is much larger than the phase velocity of the waves. The electron could thus be expected to quickly adjust to the electric potential $\phi^{\prime}$ built in a plasma, $\delta n_{e} / n_{0} \approx e \phi^{\prime} / T_{e}$ with $T_{e}=$ const. However, this is the electric potential existing in a fluid element drifting with the $E \times B$ velocity. Such an electric potential is different from the electric potential $\phi$ measured in the lab frame, therefore, the above formula is not very helpful. Instead, we express the pressure as $p_{e}=n T_{e}$, and use Eq. 5 for the electron density. We notice that in this equation, the magnetic fluctuations $\delta B_{z} / B_{0}$ are smaller than $\delta n / n_{0}$ in a low beta regime. Indeed, from the plasma momentum equation (the sum of the electron and ion momentum equations), one can derive to the leading order the (total) pressure balance condition $\nabla_{\perp} p=0$, which gives $\left(\delta B_{z} / B_{0}\right)=-\left(\beta_{e} / 2\right)\left(\delta n / n_{0}\right)$. We can, therefore, neglect the magnetic fluctuations in Eq. 5. We also neglect the electron polarization drift, and obtain

$$
\frac{\partial}{\partial t}\left(\frac{\delta n}{n_{0}}\right)+\left(\widehat{z} \times \nabla_{\perp} \phi\right) \cdot \nabla_{\perp}\left(\frac{\delta n}{n_{0}}\right)=-d_{i} \nabla_{\|} \nabla_{\perp}^{2} A_{z},
$$

which, together with Eqs. 12, 13, forms a closed system of equations for the considered case.

The dispersion relation for the linear waves in this case is:

$$
\omega^{2}=\frac{k_{z}^{2} v_{A}^{2}\left(1+k_{\perp}^{2} \rho_{s}^{2}\right)}{\left(1+k_{\perp}^{2} d_{e}^{2}\right)},
$$

where $\rho_{s}^{2}=v_{s}^{2} / \Omega_{i}^{2}$ is the ion-acoustic radius and $v_{s}^{2}=T_{e} / m_{i}$ is the ion acoustic speed. Since $\rho_{s}^{2} / d_{e}^{2}=\beta_{e} /\left(m_{e} / m_{i}\right)$, this formula shows that depending on the value of the electron beta, either the ionacoustic scale or the electron inertial scale becomes dominant.
The quadratic conservation laws for this case are the energy and generalized enstrophy:

$$
\begin{gathered}
E=\int\left[\left(\nabla_{\perp} A_{z}\right)^{2}+d_{e}^{2}\left(\nabla_{\perp}^{2} A_{z}\right)^{2}+\left(\nabla_{\perp} \phi\right)^{2}+\rho_{s}^{2}\left(\frac{\delta n}{n_{0}}\right)^{2}\right] d^{3} x \\
\Omega_{2}=\int\left(\frac{\delta n}{n_{0}}-\frac{1}{\Omega_{i}} \nabla_{\perp}^{2} \phi\right)^{2} d^{3} x .
\end{gathered}
$$

In fact, there are infinitely many conserved integrals of the form

$$
\Omega_{n}=\int\left(\frac{\delta n}{n_{0}}-\frac{1}{\Omega_{i}} \nabla_{\perp}^{2} \phi\right)^{n} d^{3} x,
$$

which simply reflects the fact that the two-dimensional $E \times B$ flow is incompressible, and the integrand in $\Omega_{n}$ is passively advected by such a flow.

\section{Case of $m_{\mathrm{e}} / \mathrm{m}_{\mathrm{i}} \ll \beta_{\mathrm{e}} \ll 1$ and $\beta_{\mathrm{i}} \lesssim 1$ (Hot Electrons and lons)}

We now consider the case of relatively high temperatures of the electrons and the ions. In this case, the ion gyroscale is not small. At scales close to the ion gyroscale, fluid-like models are generally not accurate, and one has to use full kinetic treatment. However, at larger and smaller scales one can formulate simplified models. Obviously, at hydrodynamic scales $k_{\perp}^{2} \rho_{i}^{2} \ll 1$, a good description is provided by the reduced MHD model. Here we will be interested in scales smaller than the ion gyroscale, $k_{\perp}^{2} \rho_{i}^{2} \gg 1$. In this limit, the system of equations has been derived in (Chen and Boldyrev, 2017; Passot et al., 2017; Passot et al., 2018). As can be checked later, in this case the ions can be considered hot, $\omega^{2} \ll k^{2} v_{T i}^{2}$, and non magnetized. Therefore, their density, and by quasineutrality the density of the electrons, will adjust to the electric potential existing in a plasma according to the Boltzmann law, $\delta n / n_{0} \approx-e \phi / T_{i}$. Similarly to the previous case, the magnetic intensity fluctuations can be evaluated from the momentum equation, where both the ion and the electron temperatures can be easily taken into account as both species are now hot:

$$
\left(\delta B_{z} / B_{0}\right)=-\left(\beta_{e} / 2+\beta_{i} / 2\right)\left(\delta n / n_{0}\right) \approx-\left(\beta_{i} / 2\right)\left(\delta n / n_{0}\right) .
$$

We can now remove the density and magnetic field fluctuations in the electron Eqs. 5, 12 in favor of the electric potential, and obtain:

$$
\begin{gathered}
\frac{\partial}{\partial t}\left(1+\frac{2}{\beta_{i}}-d_{e}^{2} \nabla_{\perp}^{2}\right) \phi=v_{A} d_{i}^{2} \nabla_{\|} \nabla_{\perp}^{2} A_{z} \\
\frac{\partial}{\partial t}\left(1-d_{e}^{2} \nabla_{\perp}^{2}\right) A_{z}+\left(\widehat{z} \times \nabla_{\perp} \phi\right) \cdot \nabla_{\perp}\left(1-d_{e}^{2} \nabla_{\perp}^{2}\right) A_{z}=-v_{A} \frac{\partial}{\partial z} \phi
\end{gathered}
$$

The linear modes described by this system have the dispersion relation: 


$$
\omega^{2}=\frac{k_{z}^{2} v_{A}^{2} k_{\perp}^{2} d_{i}^{2}}{\left(1+k_{\perp}^{2} d_{e}^{2}\right)\left(1+2 / \beta_{i}+k_{\perp}^{2} d_{e}^{2}\right)}
$$

such modes were termed the inertial kinetic-Alfvén modes in Chen and Boldyrev (2017). A particular case of these waves, corresponding to the limit $2 / \beta_{i} \gg 1+k_{\perp}^{2} d_{e}^{2}$, has been previously analyzed in Shukla et al. (2009), Agarwal et al. (2011); such an additional constraint obviously implies a more limited region of applicability of the model, namely, $k_{\perp}^{2} \rho_{i}^{2} \ll m_{i} / m_{e}$. The considered system has two quadratic conservation laws, the energy and generalized helicity (Boldyrev and Loureiro, 2020):

$$
\begin{gathered}
E=\int\left[\phi\left(1+\frac{2}{\beta_{i}}-d_{e}^{2} \nabla_{\perp}^{2}\right) \phi-d_{i}^{2}\left(\nabla_{\perp}^{2} A_{z}\right)\left(1-d_{e}^{2} \nabla_{\perp}^{2}\right) A_{z}\right] d^{3} x \\
H=\int\left(1+\frac{2}{\beta_{i}}-d_{e}^{2} \nabla_{\perp}^{2}\right) \phi\left(1-d_{e}^{2} \nabla_{\perp}^{2}\right) A_{z} d^{3} x
\end{gathered}
$$

The derived conservation laws play an important role in turbulent cascades as well as in the formation of current sheets that may become subject to the tearing instability and magnetic reconnection (e.g., Boldyrev and Loureiro, 2019; Vega et al., 2020). Interestingly, this system of equations turns out to be rather universal. It is structurally identical to the system describing the nonlinear whistler modes at sub-ion scales (Chen and Boldyrev, 2017), moreover, at scales $k_{\perp}^{2} d_{e}^{2} \gg 1+2 / \beta_{i}$ it is also applicable to a nonrelativistic pair plasma (Loureiro and Boldyrev, 2018) as well as to rapidly rotating non-conducting fluids, see, e.g., (Milanese et al., 2020).

\section{CONCLUSION}

We have described several physical models of nonlinear plasma dynamics at low electron beta, which are relevant for space physics applications ranging from the Earth's magnetosphere to the magnetosheath to the solar corona. These models may be helpful

\section{REFERENCES}

Agarwal, P., Varma, P., and Tiwari, M. S. (2011). Study of inertial kinetic Alfven waves around cusp region. Planet. Space Sci. 59, 306-311. doi:10.1016/j.pss.2010.11.006

Bale, S. D., Goetz, K., Harvey, P. R., Turin, P., Bonnell, J. W., Dudok de Wit, T., et al. (2016). The FIELDS instrument suite for solar Probe plus. Measuring the coronal plasma and magnetic field, plasma waves and turbulence, and radio signatures of solar transients. Space Sci. Rev. 204, 49-82. doi:10.1007/s11214-016-0244-5

Biskamp, D. (2003). Magnetohydrodynamic turbulence. Cambridge, United Kingdom: Cambridge University Press.

Boldyrev, S., Chen, C. H. K., Xia, Q., and Zhdankin, V. (2015). Spectral breaks of alfvénic turbulence in a collisionless plasma. Astrophys. J. 806, 238. doi:10.1088/ 0004-637X/806/2/238

Boldyrev, S., and Loureiro, N. F. (2019). Role of reconnection in inertial kinetic-AlfvÃ@n turbulence. Phys. Rev. Res. 1, 012006. doi:10.1103/PhysRevResearch.1.012006

Boldyrev, S., and Loureiro, N. F. (2020). Tearing instability in Alfvã $\odot n$ and kineticAlfvÃ@n turbulence. J. Geophys. Res. Space Phys. 125, e2020JA028185. doi:10. 1029/2020JA028185

Camargo, S. J., Scott, B. D., and Biskamp, D. (1996). The influence of magnetic fluctuations on collisional drift-wave turbulence. Phys. Plasmas 3, 3912-3931. doi:10.1063/1.871580 for understanding turbulent cascades (that are generally nontrivial in the presence of two conserved quantities (Loureiro and Boldyrev, 2018; Milanese et al., 2020), processes of magnetic reconnection (e.g., Boldyrev and Loureiro, 2019; Loureiro and Boldyrev, 2020), and other linear and nonlinear wave phenomena. Our fluid-like models do not include dissipation effects, like Landau damping, that cannot be rigorously treated in fluid-like models and that require kinetic approach (e.g., Chen et al., 2019; Horvath et al., 2020). The kinetic dissipation effects are especially relevant when the scales of fluctuations approach the gyroscales of plasma species or when the phase velocities of the waves are comparable to the thermal velocities of the particles, see, for instance the kinetic treatment developed for the case $\beta_{e} \sim m_{e} / m_{i}$ in Zocco and Schekochihin (2011). However, it should be noted that the ordering assumed in our models implies that the linear and nonlinear terms are on the same order (the co-called critical balance condition), which means that dissipative kinetic terms may be included as linear terms in our equations (e.g., Li et al., 2016; Passot et al., 2017; Passot et al., 2018), which should not qualitatively alter the nonlinear dynamics captured by the discussed models.

\section{AUTHOR CONTRIBUTIONS}

$\mathrm{SB}, \mathrm{NL}$, and VR performed the research; SB wrote the paper.

\section{FUNDING}

The work of SB was partly supported by the NSF under Grant Nos. NSF PHY-1707272 and NSF PHY-2010098, by the NASA under Grant No. NASA 80NSSC18K0646, and by DOE Grant No. DESC0018266. NFL was partially funded by NSF CAREER Award No. 1654168 and by the NSF-DOE Partnership in Basic Plasma Science and Engineering, Award No. PHY-2010136. VR was partially supported by DOE Grant No. DE-SC0019315.

Chen, C. H. K., Bale, S. D., Bonnell, J. W., Borovikov, D., Bowen, T. A., Burgess, D., et al. (2020). The evolution and role of solar wind turbulence in the inner heliosphere. Astrophy. J. Suppl. 246, 53. doi:10.3847/1538-4365/ab60a3

Chen, C. H., and Boldyrev, S. (2017). Nature of kinetic scale turbulence in the earth's magnetosheath. Astrophy. J. 842, 122. doi:10.3847/1538-4357/aa74e0

Chen, C. H. K., Klein, K. G., and Howes, G. G. (2019). Evidence for electron landau damping in space plasma turbulence. Nat. Commun. 10, 740. doi:10.1038/ s41467-019-08435-3

Chen, C. H. K., Leung, L., Boldyrev, S., Maruca, B. A., and Bale, S. D. (2014). Ionscale spectral break of solar wind turbulence at high and low beta. Geophys. Res. Lett. 41, 8081-8088. doi:10.1002/2014GL062009

Chen, C. H. K. (2016). Recent progress in astrophysical plasma turbulence from solar wind observations. J. Plasma Phys. 82, 121. doi:10.1017/S0022377816001124

Cranmer, S. R., Matthaeus, W. H., Breech, B. A., and Kasper, J. C. (2009). Empirical constraints on proton and electron heating in the fast solar wind. Astrophy. J. 702, 1604-1614. doi:10.1088/0004-637X/702/2/1604

Bale, S. D., Badman, S. T., Bonnell, J. W., Bowen, T. A., Burgess, D., Case, A. W., et al. (2019). Highly structured slow solar wind emerging from an equatorial coronal hole. Nature 576, 237-242. doi:10.1038/s41586-019-1818-7

Franci, L., Stawarz, J. E., Papini, E., Hellinger, P., Nakamura, T., Burgess, D., et al. (2020). Modeling MMS observations at the earth's magnetopause with hybrid 
simulations of alfvénic turbulence. Astrophy. J. 898, 175. doi:10.3847/15384357/ab9a47

Ghavamian, P., Schwartz, S. J., Mitchell, J., Masters, A., and Laming, J. M. (2013). Electronion temperature equilibration in collisionless shocks: the supernova remnant-solar wind connection. Space Sci. Rev. 178, 633-663. doi:10.1007/s11214-013-9999-0

Goldreich, P., and Sridhar, S. (1995). Toward a theory of interstellar turbulence. 2: strong alfvenic turbulence. Astrophy. J. 438, 763-775. doi:10.1086/175121

Grošelj, D. (2019). Thesis: fully kinetic simulations of microscale turbulence is space and astrophysical plasmas. MS dissertation. Munich: Ludwig-MaximiliansUniversität München.

Horvath, S. A., Howes, G. G., and McCubbin, A. J. (2020). Electron landau damping of kinetic Alfvén waves in simulated magnetosheath turbulence. arXiv e-printsarXiv:2009.05010.

Kadomtsev, B. B., and Pogutse, O. P. (1974). Nonlinear helical perturbations of a plasma in the tokamak. Soviet J. Exp. Theor. Phys. 38, 283-290.

Kasper, J. C., Bale, S. D., Belcher, J. W., Berthomier, M., Case, A. W., Chandran, B. D. G., et al. (2019). Alfvénic velocity spikes and rotational flows in the near-Sun solar wind. Nature 576, 1-4. doi:10.1038/s41586-019-1813-z

Li, T. C., Howes, G. G., Klein, K. G., and TenBarge, J. M. (2016). Energy dissipation and Landau damping in two and three-dimensional plasma turbulence. Astrophy. J. lett. 832, L24. doi:10.3847/2041-8205/832/2/L24

Loureiro, N. F., and Boldyrev, S. (2020). Nonlinear reconnection in magnetized turbulence. Astrophy. J. 890, 55. doi:10.3847/1538-4357/ab6a95

Loureiro, N. F., and Boldyrev, S. (2018). Turbulence in magnetized pair plasmas. Astrophy. J. lett. 866, L14. doi:10.3847/2041-8213/aae483

Milanese, L. M., Loureiro, N. F., Daschner, M., and Boldyrev, S. (2020). Dynamic phase alignment in inertial Alfvén turbulence. Phys. Rev. Lett. 125, 265101. doi:10.1103/PhysRevLett.125.265101

Passot, T., Sulem, P. L., and Tassi, E. (2017). Electron-scale reduced fluid models with gyroviscous effects. J. Plasma Phys. 83, 715830402. doi:10.1017/S0022377817000514

Passot, T., Sulem, P. L., and Tassi, E. (2018). Gyrofluid modeling and phenomenology of low- $\beta_{\mathrm{e}}$ Alfvén wave turbulence. Phys. Plasmas 25, 042107. doi:10.1063/1.5022528

Perez, J. C., and Boldyrev, S. (2010). Numerical simulations of imbalanced strong magnetohydrodynamic turbulence. Astrophy. J. lett. 710, L63-L66. doi:10.1088/ 2041-8205/710/1/L63

Phan, T. D., Eastwood, J. P., Shay, M. A., Drake, J. F., Sonnerup, B. U. Ö., Fujimoto, M., et al. (2018). Electron magnetic reconnection without ion coupling in Earth's turbulent magnetosheath. Nature 557, 202-206. doi:10.1038/s41586-018-0091-5

Quataert, E. (1998). Particle heating by Alfvénic turbulence in hot accretion flows. Astrophy. J. 500, 978-991. doi:10.1086/305770

Roytershteyn, V., Boldyrev, S., Delzanno, G. L., Chen, C. H. K., Grošelj, D., and Loureiro, N. F. (2019). Numerical study of inertial kinetic-Alfvén turbulence. Astrophy. J. 870, 103. doi:10.3847/1538-4357/aaf288

Schekochihin, A. A., Cowley, S. C., Dorland, W., Hammett, G. W., Howes, G. G., Quataert, E., et al. (2009). Astrophysical gyrokinetics: kinetic and fluid turbulent cascades in magnetized weakly collisional plasmas. Astrophy. J. Supp. 182, 310-377. doi:10.1088/0067-0049/182/1/310

Servidio, S., Valentini, F., Califano, F., and Veltri, P. (2012). Local kinetic effects in two-dimensional plasma turbulence. Phys. Rev. Lett. 108, 045001. doi:10.1103/ PhysRevLett.108.045001

Shebalin, J. V., Matthaeus, W. H., and Montgomery, D. (1983). Anisotropy in MHD turbulence due to a mean magnetic field. J. Plasma Phys. 29, 525-547. doi:10.1017/S0022377800000933

Shukla, N., Varma, P., and Tiwari, M. S. (2009). Study of kinetic Alfven wave in inertial regime. Indian J. Pure Appl. Phys. 47, 350-355.

Strauss, H. R. (1976). Nonlinear, three-dimensional magnetohydrodynamics of noncircular tokamaks. Phys. Fluids 19, 134-140. doi:10.1063/1.861310

Štverák, Š., Trávníček, P. M., and Hellinger, P. (2015). Electron energetics in the expanding solar wind via Helios observations. J. Geophys. Res. 120, 8177-8193. doi:10.1002/2015JA021368

Terry, P. W., McKay, C., and Fernandez, E. (2001). The role of electron density in magnetic turbulence. Phys. Plasmas 8, 2707-2721. doi:10.1063/1. 1362531

Tobias, S. M., Cattaneo, F., and Boldyrev, S. (2013). "Ten chapters in turbulence," in MHD dynamos and turbulence. Editors P. A. Davidson, Y. Kaneda, and K. R. Sreenivasan (Cambridge: Cambridge University Press), 351-404.

Valentini, F., Vásconez, C. L., Pezzi, O., Servidio, S., Malara, F., and Pucci, F. (2017). Transition to kinetic turbulence at proton scales driven by largeamplitude kinetic Alfvén fluctuations. Astron. Astrophys. 599, A8. doi:10. 1051/0004-6361/201629240

Vega, C., Roytershteyn, V., Delzanno, G. L., and Boldyrev, S. (2020). Electron-only reconnection in kinetic-alfvén turbulence. Astrophys. J. Lett. 893, L10. doi:10. 3847/2041-8213/ab7eba

Vink, J., Broersen, S., Bykov, A., and Gabici, S. (2015). On the electron-ion temperature ratio established by collisionless shocks. Astron. Astrophys. 579, A13. doi:10.1051/0004-6361/201424612

Zocco, A., and Schekochihin, A. A. (2011). Reduced fluid-kinetic equations for low-frequency dynamics, magnetic reconnection, and electron heating in lowbeta plasmas. Phys. Plasmas 18, 102309-102324. doi:10.1063/1.3628639

Conflict of Interest: The authors declare that the research was conducted in the absence of any commercial or financial relationships that could be construed as a potential conflict of interest.

Copyright (c) 2021 Boldyrev, Loureiro and Roytershteyn. This is an open-access article distributed under the terms of the Creative Commons Attribution License (CC $B Y)$. The use, distribution or reproduction in other forums is permitted, provided the original author(s) and the copyright owner(s) are credited and that the original publication in this journal is cited, in accordance with accepted academic practice. No use, distribution or reproduction is permitted which does not comply with these terms. 